Instituto Internacional de Investigación y Desarrollo Tecnológico Educativo INDTEC, C.A.

DOI: https://doi.org/10.29394/Scientific.issn.2542-2987.2020.5.18.6.120-136

OAI-PMH: http://www.indteca.com/ojs/index.php/Revista Scientific/oai

Artículo Original / Original Article

\title{
Los MOOC: Una alternativa para la formación continua
}

Autores: Lourdes Normandi Atiaja Atiaja Universidad de las Fuerzas Armadas, ESPE

Inatiaja@espe.edu.ec

Sangolquí, Ecuador

https://orcid.org/0000-0003-0043-1890

Andrés García Martínez

Universidad de la Habana, UH

agarcia@cepes.uh.cu

La Habana, Cuba

https://orcid.org/0000-0001-7782-8904

Resumen

Los avances de las Tecnologías de Información y Comunicación (TIC) aplicadas en la educación, sin duda alguna, han dado lugar a nuevas formas de aprendizajes flexibles, abiertas y gratuitas o a bajo costo como los MOOC "Massive Open Online Courses", Cursos en línea masivos y abiertos, que potencian la educación inclusiva. En este sentido el objetivo principal de este trabajo es analizar como los MOOC son un potencial para para la formación continua, cuáles son los alcances y retos que enfrentan estos cursos, a través de un estudio descriptivo y la aplicación de métodos histórico-lógicos, de análisis y síntesis y finalmente la generalización-abstracción, en base a importantes fuentes bibliográficas primarias y secundarias, con el propósito que las instituciones educativas vinculen a los $\mathrm{MOOC}$, como un recurso para la formación continua que tanto demandan los profesionales, a fin de actualizar, renovar o alcanzar nuevos conocimientos, competencias 0 habilidades y ser más competitivos; a pesar de que existe mucho trabajo por hacer para garantizar la calidad de aprendizaje de los MOOC.

Palabras clave: tecnología de la información; formación continua; tecnología educativa.

Cómo citar este artículo:

Atiaja, L., \& García, A. (2020). Los MOOC: Una alternativa para la formación continua. Revista

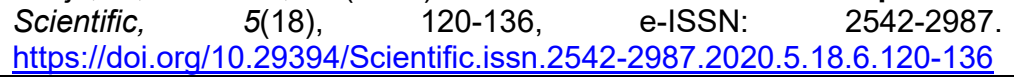

Fecha de Recepción: 24-06-2020
Fecha de Aceptación: 17-09-2020
Fecha de Publicación:

05-11-2020 
The MOOC: An alternative to continuing training

\begin{abstract}
The advances of Information and Communication Technologies (ICT) applied in education have undoubtedly given rise to new forms of flexible, open and free or low-cost learning, such as MOOC "Massive Open Online Courses". massive and open, which promote inclusive education. In this sense, the main objective of this work is to analyze how MOOCs are a potential for continuous training, what are the scope and challenges faced by these courses, through the application of historical-logical, analysis and synthesis methods and finally the generalization-abstraction, based on important primary and secondary bibliographic sources, with the purpose that educational institutions link to MOOCs, as a resource for continuous training that professionals demand so much, in order to update, renew or reach new knowledge, competencies or skills and be more competitive.
\end{abstract}

Keywords: information technology; continuous learning; educational technology.
How to cite this article:

Atiaja, L., \& García, A. (2020). The MOOC: An alternative to continuing training. Revista Scientific, 5(18), 120-136, e-ISSN: 2542-2987. Recovered from: https://doi.org/10.29394/Scientific.issn.2542$\underline{2987.2020 .5 .18 .6 .120-136}$

Date Received: 24-06-2020

Date Acceptance:

17-09-2020
Date Publication:

05-11-2020 


\section{Introducción}

Los MOOC son el resultado de la tecnología aplicada en la educación, son un fenómeno mundial, que se están convirtiendo en una nueva modalidad formativa, basada en ecologías digitales y pueden favorecer al desarrollo tecnológico, didáctico y de inclusión social y educativa; según FernándezMartínez, Martín-Padilla, Luque de la Rosa y Eguizábal-Román (2020): gracias a su carácter de cursos, masivos, abiertos y en línea.

Los MOOC aparecen por el año 2008, cuando George Siemens y Stephen Downes, lanzan por primera vez un curso denominado "Connectivism and Connective Knowledge", Conectivismo y aprendizaje conectivo, organizado por la Universidad de Manitoba (Canadá), en el que incorporaron estrategias del aprendizaje abierto basado en el conectivismo, nuevos enfoques para la interacción y las redes sociales, con una duración de 12 meses, en el que se registraron libremente, alrededor de 2.300 estudiantes.

Este tipo de cursos nacen con la idea de democratizar del conocimiento, y su concepto esta dado por las siglas del término MOOC "masive" masivo, "open" abierto, "online" en línea y "course" curso, esta modalidad de aprendizaje es relativamente nueva y hasta la presente fecha, no han alcanzado su etapa de madurez; pese a que existe una gran cantidad de literatura y divulgación científica respecto a este tema, que tanto ha llamado la atención de estudiantes, profesionales e investigadores.

En la actualidad múltiples Instituciones educativas, han adoptado a los MOOC, y más ahora, que el mundo está atravesando por una emergencia sanitaria debido a la pandemia del coronavirus (COVID-19), en donde miles de estudiantes, profesionales y trabajadores, han recurrido a los cursos masivos abiertos en línea, con la finalidad de capacitarse y adquirir conocimientos, competencias y habilidades tecnológicas, para así, poder desenvolverse en sus actividades; sobre todo en el campo educativo, los docentes se han visto obligados a capacitarse para adquirir competencias 
digitales y poder desarrollar contenidos o materiales, debido a la migración del modelo educativo tradicional hacia la virtualidad.

Es así que, los autores Benet, Sanahuja, García y Nieto (2018a): manifiestan que los MOOC son un recurso muy útil para la formación continua, porque permite el intercambio de conocimiento de una manera abierta y flexible; es decir, estos cursos favorecerían a los cientos y miles de profesionales que requieren capacitarse y de esta manera puedan acceder a una educación inclusiva.

A primera vista, los MOOC son la respuesta a esta demanda: muchas personas, en cualquier momento, libremente pueden acceder a la información, adquirirla junto a las habilidades a ella asociadas, están los medios para la actividad de aprendizaje, al alcance, supuestamente, de todos quienes cuentan con un computador con acceso a Internet y la posibilidad de conectarse a redes de aprendizaje.

En este sentido, es necesario realizar un análisis sobre como los MOOC pueden contribuir a la formación continua y cuáles son los alcances y retos que enfrentan estos cursos; de tal manera que, las instituciones educativas puedan vincular esta modalidad de aprendizaje flexible y abierto.

\section{Metodología}

El enfoque de investigación que se aplicó en el presente trabajo es de índole cualitativo y es el resultado de una revisión de la literatura de una manera sistemática y congruente, considerando el proceso de ocho etapas que especifican Okoli y Schabram (2010a):

1). Propósito de la revisión de la literatura; 2). Protocolo y formación; 3). La búsqueda de la literatura; 4) Pantalla práctica; 5). Estimación de la calidad; 6). Extracción de datos; 7). Síntesis de los estudios; y 8). Redacción de la revisión (pág. 7).

El alcance de este trabajo investigativo es descriptivo, por cuanto lo que 
se pretende es buscar los rasgos importantes y el alcance de los MOOC en la formación continua. Luego de la ejecución del proceso establecido por Okoli y Schabram (2010b): se aplicaron los métodos histórico-lógicos, de análisis y síntesis y finalmente la generalización-abstracción, en función de la literatura seleccionada en importantes fuentes bibliográficas primarias y secundarias, para de esta manera determinar el estado de los MOOC como un recurso educativo que contribuye a la formación continua que tanto demandan los profesionales; razón por la cual, múltiples instituciones educativas están incorporando dentro de su catálogo académico a los MOOC, como una alternativa para la formación continua o como una herramienta complementaria de la modalidad de estudios tradicional o a distancia.

\section{Resultados}

En la actualidad, muchos profesionales buscan programas o cursos de formación continua, movidos por la necesidad de renovar sus conocimientos, habilidades y actitudes para desarrollar sus actividades o insertarse en el mundo laboral. Indiscutiblemente la formación continua es un tema que se lleva a cabo a lo largo de la vida laboral de las personas, y es por esta razón que Pineda y Sarramona (2006): "vincula con el concepto de educación permanente y de ahí su adjetivación como «continua»" (pág. 706). Mencionando a Gil y Gallego (2016): el aprendizaje permanente es un concepto complejo y ha recibido diferentes denominaciones; es así que, en el idioma inglés existe una serie de terminologías que hacen referencia al aprendizaje permanente, como lo expresa el autor Xiaozhou (2001a): aprendizaje para toda la vida, educación permanente, educación de adultos, aprendizaje de adultos, educación en servicio, educación continua, educación permanente y educación recurrente; aunque la expresión más conocida es aprendizaje para toda la vida (Lifelong learning) y que muchos lo asocian con los MOOC.. 
Otra definición que se destaca fue establecida por la Organización para la Cooperación y el Desarrollo Económico (OCDE) que de acuerdo con Delors, et al. (1996): el aprendizaje a lo largo de la vida se considera como actividad humana que se extiende "de la cuna a la tumba". No cabe duda que el aprendizaje permanente se ha convertido en un eje transversal del ser humano. Además, se podría decir que el aprendizaje permanente, constituye un componente esencial del conocimiento; puesto que, para generar una economía, la sociedad demanda de trabajadores y profesionales competitivos, con conocimientos actualizados. Es por ello que, el desarrollo económico y la llamada sociedad digital dependerán en gran parte del avance del aprendizaje permanente; así como también del factor establecido por Xiaozhou (2001b): de como el aprendizaje se integre en el sistema formal y no formal de educación.

En este contexto, los autores de esta investigación, establecen la siguiente definición, la formación continua es un proceso a lo largo de toda la vida que tiene fines específicos como la actualización de conocimientos, competencias, habilidades, entre otros, para el desarrollo integral del ser humano y que ayuda a mejorar e innovar y dar respuesta a las necesidades productivas y de servicios generadas, por la dinámica de la ciencia, tecnología y sociedad.

Por tanto, es importante que las universidades como parte de su responsabilidad social, provean a la sociedad de herramientas o recursos que faciliten la formación continua bajo una modalidad de aprendizaje en línea y flexible como los MOOC. Señalando a Bates (2015): los MOOC son una buena contribución a la educación continua, pese a que requiere de una reingeniería. En este sentido, es necesario que las instituciones educativas adopten nuevas estrategias, metodologías para mejorar la calidad de los MOOC, de tal manera que este tipo de cursos, constituyan una herramienta de enseñanza aprendizaje dentro de la formación formal, informal y no formal y potencien la 
Educación Superior.

Es necesario resaltar que estos cursos cada vez van ganando espacio y convirtiéndose en una alternativa para la formación continua; por lo que se considera, que son una herramienta complementaria para la educación tradicional. Como dice Aguado (2020): Ios MOOC, representan uno de los máximos exponentes en el uso de la tecnología en la educación y constituyen un complemento de la formación presencial y a distancia, dado el interés del alumnado por formas de aprendizajes mixtas; además en este tipo de cursos, los docentes pueden emplear diferentes metodologías innovadoras para el proceso de enseñanza aprendizaje.

Las Universidades, últimamente han lanzado de una manera desenfrenada a la producción de MOOC, en este sentido, Luna y GonzálezFlores (2020): exponen que desde el año 2019, existen más de 13.500 cursos, de 900 universidades, a nivel mundial que ofertan cursos en diferentes plataformas $\mathrm{MOOC}$, en la que participan millones de estudiantes, quizá el auge de estos cursos en estos últimos años, se debe a que los alumnos pueden organizar el aprendizaje a su propio ritmo y más ahora en este periodo de confinamiento debido a la pandemia del COVID-19; muchas personas buscan capacitarse en una modalidad en línea y que mejor si se trata de cursos abiertos y gratuitos o bajo costo.

Si bien es cierto, estos cursos contribuyen a una educación inclusiva, presentan una serie de problemas como son: altos índices de deserción de los participantes, la falta de credibilidad, falta de motivación, retroalimentación, entre otros. Pero su principal inconveniente radica esencialmente a lo que establecen Chen, Feng, Sun, Wu, Yang y Chen (2019): en la alta tasa de abandono, que es un problema universal, que aún no ha sido resuelto en los MOOC. Posiblemente un aspecto que motiva a esta situación, quizá se debe a que los participantes al no recibir alguna respuesta frente a sus inquietudes o preguntas, prefieren abandonar los cursos; de acuerdo a la experiencia 
participativa de los autores, en las principales plataformas MOOC como Coursera, edX, Udacity, FutureLearn, Miríadax y UniMOOC, evidenciándose un estado de abandono y soledad en este tipo de cursos.

Por otro lado, también, es comprensible esta situación; pues uno, dos, tres o cinco tutores, no pueden atender a miles de participantes; por lo tanto, este problema de los MOOC, está asociada al concepto de masividad de estos cursos; mismo que se alude a la capacidad que tiene las plataformas elearning para matricular a un número ingente de participantes y que lastimosamente como lo señalan Escudero-Nahón y Nuñez-Urbina (2020): el alcance masivo no ha fomentado ni garantizado el aprovechamiento educativo. No obstante, pese a los problemas de los MOOC que vienen arrastrando desde sus inicios, las instituciones educativas están adoptando a los MOOC, como una estrategia educativa, motivadas por diferentes intereses.

De acuerdo al informe publicado por la compañía de telecomunicaciones más importante de Europa y la quinta del mundo, la Fundación Telefónica (2015): señala que los principales motivos por los que las instituciones educativas adoptan los MOOC son:

- Ampliar el alcance de la institución y el acceso a la educación; es decir diversificar la oferta académica, rompiendo las barreras del espacio y el tiempo, a través de una formación flexible.

- Construir y mantener una marca; significa que las instituciones quieren incluir MOOC para lograr la internacionalización que les permite una mejor visibilidad ante el mundo.

- Mejorar la economía mediante la reducción de costes o el aumento de los ingresos. En este caso, varias universidades están mercantilizando la educación a través de la oferta de MOOC, a cambio de la entrega de certificados por un costo.

- Mejorar los resultados educativos, tanto para los participantes en 
OAI-PMH: http://www.indteca.com/ojs/index.php/Revista_Scientific/oai

\section{Artículo Original / Original Article}

MOOC como para los estudiantes de la modalidad presencial; puesto que ya existen algunas universidades que emplean a los MOOC como una herramienta de apoyo dentro de la modalidad presencial.

- Innovar en el proceso enseñanza aprendizaje y llevar a cabo investigaciones sobre este proceso, a través de entornos flexibles y ubicuos.

Orto de los motivos por lo que las Instituciones de Educación Superior (IES) producen MOOC, se debe a las capacidades actuales que disponen y que no son suficientes, no sólo por limitaciones de infraestructura física o técnica, recursos humanos, entre otros; sino porque en muchos países, la educación está muy lejos de las posibilidades de los potenciales estudiantes: el acceso a la educación superior es demasiado costoso, o que están en otros países, o que tienen que trabajar para la familia.

Todas estas razones, han hecho que trascienda la popularidad de los MOOC y que al momento que las universidades opten por implementar esta modalidad de enseñanza aprendizaje; es necesario que la producción de los MOOC, sean concebidos desde una visión de sistema, en el que se contemplen las dimensiones pedagógica, tecnológica, organizativa y comunicacional y por otro lado, también es necesario el replanteamiento del currículum para modelos más abiertos y flexibles; puesto que para los autores Yuan y Powell (2013): esta situación obedece al carácter masivo de los cursos; de este modo se lograría un mejor aprovechamiento de los MOOC como una alternativa de formación inclusiva, y como indica la Organización de las Naciones Unidas para la Educación, la Ciencia y la Cultura (UNESCO, 2013): una oportunidad para proporcionar una formación en diferentes contextos y la posibilidad de garantizar a la población mundial una formación a lo largo de la vida. 
Desde el punto de vista de los autores, esta modalidad de aprendizaje enfrenta retos de índole tecnológico y pedagógico; mismas que están enfocados a minimizar las falencias que vienen arrastrando desde sus inicios hasta la actualidad; para ello se considera necesario la aplicación de las siguientes acciones:

1. Alfabetizar a los participantes de cursos MOOC, especialmente en el desarrollo de un nivel adecuado de uso de las herramientas tecnológicas, para que aprendan a compartir e interactuar, crear, criticar, analizar y evaluar textos multimedia y atender las responsabilidades éticas requeridas que exigen este tipo de entornos, con el fin de que los participantes de un MOOC, puedan desenvolverse en un ambiente masivo; así como también, se requiere de una capacitación digital a los profesores para que se familiaricen con las nuevas herramientas educativas.

2. Mejorar la calidad de aprendizaje a través de altos niveles de automatización, de tal manera que permitan la optimización de tiempo del docente mediante herramientas que fomenten la escalabilidad de una necesidad (incorporar más alternativas relacionadas al seguimiento, control, notificaciones, alertas, etcétera, que faciliten el trabajo del docente, sin afectar el funcionamiento del curso), para un oportuno tratamiento de la masividad.

3. Adoptar criterios de evaluación para un aprendizaje de carácter ubicuo, que sea activo, cooperativo, colaborativo, de construcción personal y colectiva con participación en redes y comunidades de aprendizaje, auténtico, basado en valores y en una evaluación reflexiva.

4. Determinar estrategias de diseño instruccional que enriquezcan el curso, donde la analítica del aprendizaje (Learning analytics), que es otro enfoque, relativamente nuevo y que se expande rápidamente, puede proporcionar información sobre el flujo de información, como 
actividades sociales, patrones de participación y una serie de otros factores, que pueden facilitar la calidad de aprendizaje y experiencia para los estudiantes, en conformidad con lo establecido por Siemens, Rudolph y Tan (2020); la web semántica (Semantic Web), los sistemas adaptativos y una acertada selección de herramientas tecno didácticas como las redes sociales, realidad aumentada, gamificación, la realidad virtual y contenidos para dispositivos móviles, podrían jugar un papel importante, de tal manera que ayuden a identificar los requerimientos de aprendizaje de los estudiantes y dar un continuo seguimiento y tutorización de los mismos.

5. Orientar a los docentes hacia las grandes líneas de investigación como es el diseño pedagógico; resaltando la importancia de que un aprendizaje activo, permite acrecentar el compromiso de participación y mejorar la retención y la motivación por el aprendizaje.

6. Diseñar nuevas metodologías que conlleven a la reflexión y criticidad sobre la práctica y la adquisición de nuevas competencias, transferibilidad de las habilidades a contextos personales, sociales, académicos y profesionales, para que de esta manera, se pueda crear la base de un aprendizaje a lo largo de la vida (Lifelong learning); que permita explotar todas las bondades que ofrece este tipo de modalidad de aprendizaje; tomando en cuenta el señalamiento de Benet, Sanahuja, García y Nieto (2018b): se debe aprovechar la heterogeneidad de los participantes que supone una oportunidad para compartir experiencias enriquecedoras.

7. Establecer un balance entre la tecnología y la pedagogía, de acuerdo a Kop y Carroll (2011): el aprendizaje se pueda favorecer a través de la interacción y colaboración, para de esta manera lograr la construcción social del conocimiento, que es un elemento clave de los MOOC.

8. Determinar estándares que validen la calidad de aprendizaje de los 
participantes de los cursos MOOC.

Si bien es cierto, los MOOC cada vez se van diversificando y expandiendo, estos siguen arrastrando una serie de problemas de índole pedagógico; en consecuencia, existe mucho trabajo por hacer para mejorar la calidad de aprendizaje de estos cursos.

\section{Conclusiones}

La actual sociedad del conocimiento demanda de profesionales con conocimientos actualizados; generando la necesidad apremiante de un aprendizaje permanente o para toda la vida; es por ello que, muchos profesionales buscan cursos o programas de capacitación flexible y en línea, que les permita actualizar y mejorar sus conocimientos, habilidades y actitudes y así, ser más competitivos y no caer en la obsolescencia laboral.

Los MOOC son una evolución del aprendizaje electrónico, resultado de la tecnología aplicada en la educación que facilitan un aprendizaje en línea, flexible, gratuitos o a bajo costo, a los cuales los estudiantes pueden acceder a través de un computador con una conexión a Internet.

Los MOOC han transformado en un interés más amplio por el aprendizaje en línea y representan una alternativa para la formación continua; además, constituyen un potencial para contribuir a satisfacer necesidades de los profesionales para actualizar, renovar o alcanzar nuevos conocimientos, competencias o habilidades, que tanto demanda la sociedad del conocimiento.

Es preciso efectuar una reconstrucción de los $\mathrm{MOOC}$, considerando la superación de los diferentes retos de índole pedagógico y tecnológico que enfrentan los cursos masivos abiertos en línea, para que de esta manera puedan mejorar la calidad de enseñanza aprendizaje de estos cursos. 


\section{Referencias}

Aguado, J. (2020). Los MOOC: ¿sustituto o complemento de la formación tradicional?. Revista Tecnología, Ciencia y Educación, (16), 41-62, eISSN: 2444-2887. Recuperado de:

https://dialnet.unirioja.es/servlet/articulo?codigo $=7492333$

Bates, A. (2015). Teaching in a Digital Age: Guidelines for Designing Teaching and Learning. ISBN: 978-0-9952692-0-0. Vancouver BC, Canadá, Estados Unidos: Tony Bates Associates Ltd.

Benet, A., Sanahuja, A., García, I., \& Nieto, R. (2018a,b). Nuevos horizontes formativos: una experiencia del MOOC como recurso en la formación continua. Apertura, 10(1), 88-103, e-ISSN: 2007-1094. Recuperado de: http://dx.doi.org/10.32870/Ap.v10n1.1151

Chen, J., Feng, J., Sun, X., Wu, N., Yang, Z., \& Chen, S. (2019). MOOC Dropout Prediction Using a Hybrid Algorithm Based on Decision Tree and Extreme Learning Machine. Mathematical Problems in Engineering, 2019, 1-11, ISSN: 1024-123X. Recovered from: https://doi.org/10.1155/2019/8404653

Delors, J., Al Mufti, I., Amagi, I., Carneiro, R., Chung, F., Geremek, B., ... Nanzhao, Z. (1996). La educación encierra un tesoro. Informe a la UNESCO de la Comisión Internacional sobre la Educación para el Siglo XXI. Compendio, ISBN: 92-3-303274-4. París, Francia: Santillana, Ediciones UNESCO.

Escudero-Nahón, A., \& Nuñez-Urbina, A. (2020). Análisis crítico al término «masivo» en los MOOC: una Cartografía Conceptual. EDMETIC, 9(1), 188-212, e-ISSN: 2254-0059. Recuperado de:

https://doi.org/10.21071/edmetic.v9i1.12252

Fernández-Martínez, M., Martín-Padilla, A., Luque de la Rosa, A., \& EguizábalRomán, I. (2020). La tecnología en el ámbito educativo ante el COVID: una apuesta por los MOOC como estrategia formativa en el 
contexto universitario. IJERI: International Journal of Educational

Research and Innovation, (15), 130-142, e-ISSN: 2386-4303.

Recuperado de: https://doi.org/10.46661/ijeri.5133

Fundación Telefónica (2015). Los MOOC en la educación del futuro: La

digitalización de la formación. ISBN: 978-84-08-13942-3. España:

Fundación Telefónica, Editorial Ariel, S.A.

Gil, A., \& Gallego, D. (2016). La realización de formación continua desde la perspectiva de la organización de aprendizaje. Educar, 52(1), 107126, e-ISSN: 2014-8801. Recuperado de:

http://dx.doi.org/10.5565/rev/educar.701

Kop, R., \& Carroll, F. (2011). Cloud Computing and Creativity: Learning on a Massive Open Online Course. EURODL: European Journal of Open, Distance and E-Learning, Special Issue on Creativity and OER, 1-11, eISSN: 1027-5207. Recovered from:

https://www.eurodl.org/materials/special/2011/Kopp Carroll.pdf

Luna, V., \& González-Flores, P. (2020). Transformaciones en educación médica: innovaciones en la evaluación de los aprendizajes y avances tecnológicos (parte 2). Investigación en Educación Médica, 9(34), 87-99, e-ISSN: 2007-5057. Recuperado de:

https://doi.org/10.22201/facmed.20075057e.2020.34.20220

Okoli, C., \& Schabram, K. (2010a,b). A Guide to Conducting a Systematic Literature Review of Information Systems Research. Philosophy \& Methodology of Economics eJournal, Available at SSRN. Recovered from: https://dx.doi.org/10.2139/ssrn.1954824

Pineda, P., \& Sarramona, J. (2006). El nuevo modelo de formación continua en España: balance de un año de cambios. Revista de Educación, 341, 705-736, e-ISSN: 1988-592X. Recuperado de: http://www.educacionyfp.gob.es/revista-de-educacion/numeros-revistaeducacion/numeros-anteriores/2006/re341/re341-29.html 
Siemens, G., Rudolph, J., \& Tan, S. (2020). "As human beings, we cannot not learn". An interview with Professor George Siemens on connectivism, MOOCs and learning analytics. Journal of Applied Learning \& Teaching, 3(1), 108-119, e-ISSN: 2591-801X. Recovered from: https://doi.org/10.37074/jalt.2020.3.1.15

UNESCO (2013). Directrices para las políticas de aprendizaje móvil. ISBN: 978-92-3-001145-1. Paris, Francia: Organización de las Naciones Unidas para la Educación, la Ciencia y la Cultura. Recuperado de: https://unesdoc.unesco.org/ark:/48223/pf0000219662

Xiaozhou, X. (2001a,b). The role of the university in lifelong learning: perspectives from the People's Republic of China. Higher Education Policy, 14, 313-324, e-ISSN: 1740-3863. Recovered from:

https://link.springer.com/article/10.1016/S0952-8733(01)00027-7

Yuan, L., \& Powell, S. (2013). MOOCs and Open Education: Implications for Higher Education. Cetis Publications, 1-21. United Kingdom: Publication from Cetis LLP. Recovered from: https://publications.cetis.org.uk/2013/667 


\section{Lourdes Normandi Atiaja Atiaja}

e-mail: Inatiaja@espe.edu.ec

Nacida en la provincia de Cotopaxi, Ecuador, el 26 de mayo

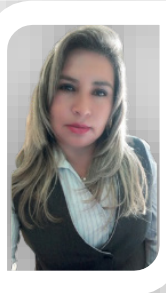
del año 1975. Profesora de la Universidad de las Fuerzas Armadas (ESPE); graduada de Ingeniera en Sistemas y Magister en Gerencia de Sistemas en la Universidad de las Fuerzas Armadas (ESPE), Ecuador; experta en E-learning; Diplomado en Competencias Docentes y Diplomado en la enseñanza del idioma inglés en el Instituto Tecnológico y de Estudios Superiores de Monterrey (ITESM); tengo una amplia participación en eventos científicos y académicos nacionales e internacionales en calidad de ponente, conferencias y ponencias, y he publicado varios artículos científicos en revistas indexadas en importantes bases de datos como Scopus, Latindex, entre otras. 


\section{Andrés García Martínez}

e-mail: agarcia@cepes.uh.cu

El contenido de este manuscrito se difunde bajo una Licencia de Creative Commons ReconocimientoNoComercial-Compartirlgual 4.0 Internacional 